\title{
Análisis de los factores de riesgo de la hipertensión arterial en Colima, México
}

\author{
Francisco Espinoza-Gómez, ${ }^{1}$ Gabriel Ceja-Espíritu, ${ }^{1,2}$ Benjamín \\ Trujillo-Hernández, ${ }^{2}$ Tania Uribe-Araiza, ${ }^{1}$ Pilar Abarca-de Hoyos ${ }^{1}$ \\ y Diana P. Flores-Vázquez ${ }^{1}$
}

Forma de citar Espinoza-Gómez F, Ceja-Espíritu G, Trujillo-Hernández B, Uribe-Araiza T, Abarca-de Hoyos P, FloresVázquez DP. Análisis de los factores de riesgo de la hipertensión arterial en Colima, México. Rev Panam Salud Publica. 2004:16(6):402-7.

RESUMEN Objetivo. Evaluar la posible asociación de la edad, el sexo, el sobrepeso, los antecedentes familiares de hipertensión arterial (HTA), el alcoholismo y el sedentarismo con la HTA en la población adulta de la ciudad de Colima, México.

Métodos. Estudio transversal analítico de base poblacional. Se aplicó una encuesta estructurada a 280 adultos mayores de 30 años que residian en la ciudad mexicana de Colima en 2001 y 2002. Las variables estudiadas fueron el sexo, la edad, el peso, la talla, los antecedentes familiares de HTA, la práctica de ejercicio físico, el tabaquismo y el consumo de alcohol. La presión arterial (PA) se midió por el método auscultatorio. Las mediciones limítrofes o dudosas se repitieron cuatro o cinco días después. Se consideró que había HTA cuando la PA sistólica era $\geq 140 \mathrm{~mm} \mathrm{Hg}$ y la presión arterial diastólica era $\geq 90 \mathrm{~mm} \mathrm{Hg}$, o la persona estaba bajo tratamiento antihipertensivo. Se calcularon las razones de posibilidades (odds ratios, $R P$ ) de las variables estudiadas y sus intervalos de confianza de 95\% (IC95\%). La asociación entre las variables y la HTA se estimó mediante regresión logística y la interacción mediante el coeficiente de productos de interacción.

Resultados. La prevalencia bruta de HTA fue de 28,6\%. La prevalencia fue mayor en hombres que en mujeres (42,1\% frente a 19,2\%, respectivamente; RP $=3,04 ;$ IC95\%: 1,8 a 5,2) y en personas mayores de 49 años que en personas de 30 a 49 años (36,8\% frente a 21,9\%, respectivamente; $R P=2,07$; IC95\%: 1,22 a 3,50). Los antecedentes familiares de HTA y el sobrepeso mostraron asociación con la HTA, mientras que la práctica de ejercicio físico tuvo un efecto protector $(R P=0,45 ; 0,23$ a 0,86). Se encontró interacción entre la HTA y la edad ( $\geq 50$ años), los antecedentes familiares de HTA, el sobrepeso y la práctica de ejercicio físico, particularmente en mujeres.

Conclusiones. La prevalencia de HTA en Colima es muy semejante a la encontrada a nivel nacional en México. Su fuerte asociación con el sexo masculino, independientemente de las otras variables, resalta la necesidad de promover campañas preventivas más enfocadas en los hombres.

Palabras clave Hipertensión, factores de riesgo, México.

Facultad de Medicina, Universidad de Colima, Colima, Col., México. La correspondencia debe ser dirigida a Francisco Espinoza Gómez, Facultad de Medicina, Universidad de Colima, Ave. Universidad 333, CP 28040, Colima, Col., México. Correo electrónico: fespin@cgic.ucol.mx
La hipertensión arterial (HTA) es uno de los problemas de salud más im-

\footnotetext{
2 Unidad de Investigación en Epidemiología Clínica, Hospital General de Zona y Medicina Familiar No. 1, IMSS, Colima, Col., México.
}

portantes en la actualidad por ser el principal factor de riesgo de la enfermedad coronaria, que a su vez constituye la primera causa de muerte a escala mundial (1). La creciente expan- 
sión del estilo de vida urbano característico de los países industrializados, con una mayor tendencia al sedentarismo y a la obesidad, ha favorecido el aumento de la prevalencia de HTA y de las complicaciones cardiovasculares derivadas de este trastorno. Este aumento de la prevalencia de HTA en el mundo se debe al envejecimiento de la población y a la ampliación de los criterios de diagnóstico empleados (2), lo que ha contribuido a que se registren tasas cada vez más elevadas, especialmente en las comunidades urbanas.

Se calcula que la prevalencia de HTA en la población mexicana mayor de 30 años es de $30 \%$ (3). Se ha observado una tendencia al aumento de la prevalencia de esta enfermedad en México como parte del fenómeno denominado "transición epidemiológica", en el cual las enfermedades crónicas y degenerativas van desplazando de los primeros lugares de morbilidad y mortalidad a las enfermedades infectocontagiosas.

Los factores de riesgo asociados con el desarrollo de la HTA son el sobrepeso (4), el sedentarismo (5), la edad y el sexo masculino (6). También se han propuesto como factores de riesgo de HTA el nivel socioeconómico bajo, el alcoholismo, el tabaquismo y los antecedentes familiares de HTA (7-9). Sin embargo, su grado de asociación no ha sido definido claramente, sobre todo con relación a los casos de HTA leve o limítrofe (10). Por otra parte, el peso relativo que tiene cada uno de esos factores de riesgo y la interacción que pudiera existir entre ellos han sido poco estudiados $(6,10)$. En un análisis de los factores de riesgo de HTA en México se encontró que las mujeres jóvenes no obesas tenían una menor tendencia a padecer de HTA que otros grupos de adultos, y que a medida que aumentaba la edad, las tasas de HTA en hombres y mujeres se equilibraban (7). Sin embargo, en ese estudio no se exploraron otras variables que pueden ser factores de confusión, como los antecedentes familiares de HTA, el sedentarismo, el tabaquismo y el alcoholismo.

El reconocimiento de la contribución relativa de cada uno de los factores mencionados al desarrollo de la HTA, sobre todo en los casos de HTA limítrofe o leve, es crucial para poder planear estrategias preventivas más eficientes, orientadas a los grupos de población más vulnerables $(2,9)$. La ciudad de Colima, en México, es una comunidad en proceso de transición del medio rural al urbano, caracterizada por una frecuencia creciente de enfermedades cardiovasculares asociadas con la HTA, por lo que constituye un escenario adecuado para estudiar los factores de riesgo de HTA en este tipo de población.

El objetivo del presente trabajo fue evaluar la posible asociación de la edad, el sexo, el sobrepeso, los antecedentes familiares de HTA, el alcoholismo y el sedentarismo con la HTA en la población adulta de la ciudad de Colima, México.

\section{MATERIALES Y MÉTODOS}

Para este estudio transversal analítico de base poblacional se aplicó una encuesta estructurada y se midió la presión arterial de 280 adultos mayores de 30 años que residían en la ciudad mexicana de Colima (municipios Colima y Villa de Álvarez, que agrupan a 210000 habitantes) durante los años 2001 y 2002. Esta ciudad es la capital del estado de Colima y se encuentra ubicada en la costa occidental del país (11).

Para el muestreo se dividió el plano de la ciudad en cuadrantes de aproximadamente 1 hectárea y a cada uno se le asignó un número que posteriormente fue seleccionado al azar por el programa informático EPIDAT 3.0. En cada cuadrante se seleccionó de manera aleatoria un número de adultos proporcional al número de habitantes de cada cuadrante, según los datos demográficos de las Áreas Geográficas Básicas (AGEB) de Colima (11). En la encuesta se tomaron los siguientes datos: sexo, edad, peso (en kilogramos), talla (en centímetros), antecedentes familiares de HTA, práctica habitual del ejercicio físico, tabaquismo y consumo de alcohol (tipo de bebida e ingesta promedio semanal). La presión arterial (PA) se midió en reposo en tres ocasiones por el método auscultatorio, con un esfigmomanómetro de mercurio, según las recomendaciones de Perloff modificadas (12). En caso de valores de PA limítrofes o dudosos, se repitieron las mediciones cuatro o cinco días después. Las mediciones de PA estuvieron a cargo de personal previamente entrenado (13).

Para el análisis estadístico, las variables se consideraron de forma dicotómica de la siguiente manera:

a. Edad: 50 años o más $=1$, de 30 a 49 años $=0$

b. Sexo: masculino $=1$, femenino $=0$

c. Sobrepeso (índice de masa corpo$\left.\mathrm{ral}^{3}[\mathrm{IMC}] \geq 27,3\right)$ : sí $=1$, no $=0$

d. Práctica habitual de ejercicio físico (más de 4 horas a la semana de trote, caminata, aeróbicos, gimnasia u otro deporte durante más de 6 meses): sí = 1 , no $=0$

e. Antecedentes familiares de HTA (alguno de los padres con diagnóstico de o tratamiento por HTA): sí = 1, no $=0$

f. Tabaquismo (consumo de 10 cigarrillos o más diarios durante más de 5 años): sí $=1$, no $=0$

g. Alcoholismo (ingesta de más de 100 $\mathrm{mL}$ de bebidas alcohólicas a la semana durante los últimos 6 meses): sí $=1$, no $=0$

La PA (variable dependiente) se expresó de manera dicotómica nominal y de acuerdo con las recomendaciones de la Organización Mundial de la Salud (OMS) (2): HTA (presión arterial sistólica $\geq 140$ y presión arterial diastólica $\geq 90 \mathrm{~mm} \mathrm{Hg}$ ) = 1 y normotensión (PA sistólica $<129$ y diastólica $<89 \mathrm{~mm}$ $\mathrm{Hg})=0$. Las personas con diagnóstico previo o bajo tratamiento antihipertensivo también se consideraron hipertensas, aun cuando las mediciones de PA realizadas fueran normales.

El análisis estadístico se realizó mediante la prueba exacta de Fisher y la corrección de Yates (programa EPIDAT 3.0). Se calcularon las razones de posibilidades (odds ratios, RP) de las variables estudiadas y sus intervalos de confianza de 95\% (IC95\%). Para ex-

\footnotetext{
El IMC se calculó como la razón entre el peso en kilogramos y el cuadrado de la talla en metros (14).
} 
plorar la asociación entre las variables y la HTA, así como las posibles interacciones, se utilizaron la regresión logística simple y la regresión logística multifactorial con selección descendente de variables (backward). Cada modelo se ajustó por el método de máxima verosimilitud de acuerdo a los lineamientos de Newton, citados por Hosmer y Lemeshow (15). La interacción bifactorial con la HTA se verificó mediante el coeficiente de productos de interacción bifactorial (16), que consiste en construir una nueva variable mediante la multiplicación de las dos primeras expresadas en escala dicotómica (presente $=1$, ausente $=0$ ). Para estos análisis se utilizó el programa SPSS.

\section{RESULTADOS}

De los 280 participantes, 166 (59,3\%) eran mujeres y 114 (40,7\%) eran hombres. El valor promedio de la PA sistólica fue de 120,0 mm Hg (IC95\%: 118,5 a 123,4), el de la PA diastólica fue de $79,2 \mathrm{~mm} \mathrm{Hg}$ (IC95\%: 77,0 a 82,1) y el de la PA media fue de $92,8 \mathrm{~mm} \mathrm{Hg}$ (IC95\%: 91,3 a 94,3).
Del total de personas encuestadas, $174(62,1 \%)$ mostraron valores de PA $<124 / 84 \mathrm{~mm} \mathrm{Hg}$ en la primera medición (normotensos), 34 (12,1\%) presentaron valores superiores a 135/95 mm $\mathrm{Hg}$ (hipertensos) y 30 (10,7\%) manifestaron estar tomando medicamentos antihipertensivos (14 mujeres y 16 hombres). De las 42 personas con valores de PA limítrofes o dudosos, 16 (5,7\% del total) mostraron cifras elevadas (PA > $140 / 90 \mathrm{~mm} \mathrm{Hg}$ ó PA media $>95 \mathrm{~mm}$ $\mathrm{Hg}$ ) durante la segunda medición. En total, 80 personas presentaron HTA según los nuevos lineamientos de la OMS $(2,12)$, para una prevalencia de $28,6 \%$ (IC95\%: $23,3 \%$ a $34,1 \%$ ) en la población estudiada. No se encontraron diferencias significativas entre hombres y mujeres en cuanto a la edad ( $\geq$ 50 años), el sobrepeso, la práctica de ejercicio físico, el tabaquismo o los antecedentes familiares de HTA. Sin embargo, el consumo de alcohol resultó ser mayor en hombres que en mujeres $\left(\chi^{2}=5,51, R P=2,98 ; P=0,018\right)$.

La prevalencia de HTA en hombres fue significativamente superior a la encontrada en mujeres $(42,1 \%$ frente a $19,2 \%$, respectivamente; $\mathrm{RP}=3,04$;
IC95\%: 1,8 a 5,2). Las personas mayores de 49 años tuvieron una prevalencia de HTA de $36,8 \%$, superior a la encontrada en las personas de 30 a 49 años (21,9\%; RP = 2,07; IC95\%: 1,22 a 3,50 ) (cuadro 1). Los antecedentes familiares de HTA y el sobrepeso mostraron una asociación con la HTA, mientras que la práctica de ejercicio físico tuvo un efecto protector significativo (RP $=0,45$; IC95\%: 0,23 a 0,86). No se observó una asociación significativa entre el tabaquismo o la ingesta de alcohol y la HTA. En el caso del alcoholismo, solamente se consideraron para el análisis 142 casos cuya información era confiable (cuadro 1).

Según los resultados de la regresión logística multifactorial, el valor de máxima similitud para el modelo resultó altamente significativo $\left(\chi^{2}=-2,0\right)$ e indicó una franca interacción inversa entre las variables sexo masculino, edad ( $\geq 50$ años), antecedentes familiares de HTA, sobrepeso y práctica de ejercicio físico (cuadro 2). En vista del efecto inverso del sexo masculino sobre todas las interacciones, se decidió estratificar las asociaciones según el sexo y se encontró que esas variables

CUADRO 1. Asociación entre las variables exploradas y la hipertensión arterial (HTA), según la regresión logística simple. Colima, México, 2000-2001

\begin{tabular}{|c|c|c|c|c|c|c|c|c|}
\hline Variable & No. & HTA & $\%$ & Coeficiente & $P$ & $\chi^{2}$ & $\begin{array}{c}\text { Razón de } \\
\text { posibilidades }\end{array}$ & $\operatorname{IC} 95 \%{ }^{a}$ \\
\hline \multicolumn{9}{|l|}{ Sexo } \\
\hline Mujeres & 166 & 32 & 19,3 & & 1,00 & & 1,00 & \\
\hline Hombres & 114 & 48 & 42,1 & 1,11 & $<0,0001$ & 16,1 & 3,04 & $1,8-5,2$ \\
\hline \multicolumn{9}{|l|}{ Edad } \\
\hline 30-49 años & 155 & 34 & 21,9 & & 1,00 & & 1,00 & \\
\hline$\geq 50$ años & 125 & 46 & 36,8 & 0,73 & 0,006 & 7,0 & 2,07 & $1,22-3,5$ \\
\hline \multicolumn{9}{|c|}{ Práctica de ejercicio físico } \\
\hline Sí & 78 & 14 & 18,0 & $-0,79$ & 0,016 & 6,35 & 0,45 & $0,23-0,86$ \\
\hline No & 202 & 66 & 32,7 & & 1,00 & & & \\
\hline \multicolumn{9}{|l|}{ Sobrepeso } \\
\hline Sí & 99 & 40 & 40,4 & 0,87 & 0,0014 & 10,26 & 2,39 & $1,4-4,07$ \\
\hline No & 181 & 40 & 22,1 & & 1,00 & & & \\
\hline \multicolumn{9}{|l|}{ Alcoholismo ${ }^{b}$} \\
\hline Sí & 31 & 11 & 35,5 & 0,13 & 0,75 & 0,1 & 1,45 & $0,49-2,64$ \\
\hline No & 111 & 36 & 32,4 & & 1,00 & & & \\
\hline \multicolumn{9}{|l|}{ Tabaquismo } \\
\hline Sí & 73 & 22 & 30,1 & 0,1 & 0,73 & 0,11 & 1,1 & $0,61-1,98$ \\
\hline No & 207 & 58 & 28,0 & & 1,00 & & & \\
\hline \multicolumn{9}{|c|}{ Antecedentes familiares de HTA } \\
\hline Sí & 92 & 36 & 39,1 & 0,74 & 0,0067 & 7,28 & 2,1 & $1,22-3,6$ \\
\hline No & 188 & 44 & 23,4 & & 1,00 & & & \\
\hline
\end{tabular}


CUADRO 2. Asociación entre las principales variables exploradas ${ }^{\mathrm{a}}$ y la hipertensión arterial (HTA), según la regresión logística multifactorial $(n=280)$. Colima, México, 2000-2001

\begin{tabular}{lcccc}
\hline \multicolumn{1}{c}{ Variable $^{\mathrm{a}}$} & Coeficiente $^{\mathrm{b}}$ & $P$ & $\begin{array}{c}\text { Razón de } \\
\text { posibilidades }\end{array}$ & IC95\%c $^{\mathrm{c}}$ \\
\hline Sexo masculino & 1,06 & 0,003 & 2,89 & $1,63-5,12$ \\
Edad $\geq 50$ años & 0,48 & 0,096 & 1,62 & $0,91-2,87$ \\
Antecedentes familiares de HTA & 0,77 & 0,009 & 2,16 & $1,21-3,88$ \\
Práctica de ejercicio físico & $-0,7$ & 0,044 & 0,49 & $0,25-0,98$ \\
Sobrepeso & 0,63 & 0,03 & 1,87 & $1,06-3,32$ \\
\hline
\end{tabular}

a En escala dicotómica: sí $=1$, no $=0$.

${ }^{\mathrm{b}}$ Coeficiente de la interacción del modelo $=-2,0$; máxima similitud para el modelo: $\chi^{2}=39,5$; grados de libertad $=5 ; P<$ 0,0001 .

c Intervalo de confianza de $95 \%$. se mantuvieron asociadas en grado significativo con la HTA solamente en mujeres (cuadro 3).

Los resultados del análisis con dos variables mediante el coeficiente de productos de interacción (16) confirmaron que las interacciones entre las combinaciones de las variables asociadas con la HTA solo fueron significativas en mujeres. Se deben resaltar las interacciones encontradas con relación a la HTA entre el sedentarismo y la edad, y entre el sobrepeso y los antecedentes familiares de HTA (cuadro 4).

\section{DISCUSIÓN}

Aunque por su diseño analítico, el objetivo principal del presente estudio no fue calcular la prevalencia de HTA en adultos de Colima, el valor encontrado en la población estudiada $(26,8 \%)$ puede reflejar la prevalencia real de HTA en la población de adultos en esa región del país. Este resultado es similar al obtenido en una encuesta nacional realizada recientemente en México, que informó de una prevalencia de 30\% en personas mayores de 20 años (3) y refleja un notable incremento con respecto a las prevalencias de HTA de $3,5 \%$ a $12 \%$ encontradas en décadas anteriores (17). Estas diferencias se podrían explicar por la creciente urbanización en México durante los últimos años, que lleva a un estilo de vida más sedentario y a hábitos alimentarios menos saludables. Sin embargo, también debe tomarse en cuenta que los
CUADRO 3. Variables con mayor influencia sobre la hipertensión arterial (HTA), según la regresión logística estratificada por sexo

\begin{tabular}{|c|c|c|c|c|c|}
\hline Variables & Coeficiente & $\chi^{2}$ & $P$ & $\begin{array}{c}\text { Razón de } \\
\text { posibilidades }\end{array}$ & IC95\%a \\
\hline \multicolumn{6}{|l|}{ Sobrepeso } \\
\hline Mujeres & 1,12 & 7,75 & 0,0053 & 3,08 & $1,39-6,8$ \\
\hline Hombres & 0,54 & 1,96 & 0,16 & 1,72 & $0,95-3,43$ \\
\hline \multicolumn{6}{|l|}{ Edad $^{b}$} \\
\hline Mujeres & 0,06 & 13,6 & 0,0003 & 1,06 & $1,03-1,09$ \\
\hline Hombres & $-0,002$ & 0,02 & 0,89 & 0,99 & $0,96-1,02$ \\
\hline \multicolumn{6}{|c|}{ Antecedentes familiares de HTA } \\
\hline Mujeres & 1,01 & 6,37 & 0,011 & 2,76 & $1,25-6,07$ \\
\hline Hombres & 0,63 & 2,44 & 0,12 & 1,88 & $0,89-3,07$ \\
\hline \multicolumn{6}{|c|}{ Práctica de ejercicio físico } \\
\hline Mujeres & $-1,23$ & 5,9 & 0,029 & 0,29 & $0,09-0,88$ \\
\hline Hombres & $-0,5$ & 1,3 & 0,26 & 0,6 & $0,25-1,44$ \\
\hline
\end{tabular}

criterios diagnósticos de HTA se han ampliado en los últimos años, según los nuevos lineamientos de la OMS (2), lo que eleva el número de personas consideradas hipertensas que no eran detectadas por los sistemas de vigilancia epidemiológica anteriores.

A pesar de que la mayor parte de los estudios realizados por encuestas abarcan a grupos de personas mas jóvenes (a partir de 20 años de edad), en el presente trabajo se entrevistó solamente a personas mayores de 30 años, ya que se consideró que la HTA en personas menores de 30 años es poco frecuente y por lo general es consecuencia de otras enfermedades, cuyos factores de riesgo son diferentes a los de la HTA esencial (2).

La mayor prevalencia de HTA encontrada en hombres en comparación con las mujeres, y en personas de mayor edad o con sobrepeso confirma lo descrito por otros autores $(2,3,5,6)$. Sin embargo, se reconoce plenamente que la prevalencia de HTA en mujeres mayores de 50 años tiende a igualarse con la de los hombres $(6,9)$, posiblemente debido a que después de la menopausia se pierde el efecto protector de los estrógenos. Según los resultados experimentales obtenidos en ratas hipertensas, esta pérdida puede deberse al agotamiento de esa hormona y al polimorfismo del gen para receptores de angiotensina I, asociado con el sexo femenino (18).

Los resultados obtenidos no confirmaron la asociación entre el consumo de alcohol y la HTA encontrada por otros autores (19), a pesar del elevado consumo de bebidas alcohólicas observado $(21,8 \%)$, especialmente en hombres, que fue el grupo con la mayor prevalencia de HTA. Es posible que la encuesta empleada careciera de la sensibilidad suficiente para detectar esa asociación, por lo que se recomienda aplicar otro tipo de formato en estudios futuros, como el propuesto por Rehm y colaboradores (20) y analizar un número mayor de personas. No obstante, es posible que de existir esa asociación, no sea tan significativa como las otras aquí encontradas. El tabaquismo también se ha identificado entre los factores de riesgo de HTA (5, 
CUADRO 4. Principales interacciones de las variables asociadas con la hipertensión arterial (HTA), mediante la regresión logística de los coeficientes de productos de interacción ${ }^{\mathrm{a}}$

\begin{tabular}{|c|c|c|c|c|c|}
\hline Variables & $\begin{array}{l}\text { Coeficiente } \\
\text { de la } \\
\text { interacción }\end{array}$ & $\chi^{2}$ & $\begin{array}{c}P \text { de la } \\
\text { interacción }\end{array}$ & $\begin{array}{c}\text { Razón de } \\
\text { posibilidades }\end{array}$ & IC95\% ${ }^{b}$ \\
\hline \multicolumn{6}{|c|}{ Sobrepeso y sedentarismo ${ }^{C}$} \\
\hline Mujeres & 1,07 & 6,55 & 0,01 & 2,94 & $1,30-6,60$ \\
\hline Hombres & 0,72 & 3,20 & 0,07 & 2,05 & $0,93-4,56$ \\
\hline \multicolumn{6}{|c|}{$\begin{array}{l}\text { Sobrepeso y antecedentes } \\
\text { familiares de HTA }\end{array}$} \\
\hline Mujeres & 1,58 & 10,64 & 0,001 & 4,87 & $1,92-12,3$ \\
\hline Hombres & 0,64 & 1,56 & 0,21 & 1,90 & $0,69-5,26$ \\
\hline \multicolumn{6}{|l|}{ Sobrepeso y edadd } \\
\hline Mujeres & 0,99 & 4,13 & 0,035 & 2,70 & $1,06-6,78$ \\
\hline Hombres & 0,71 & 2,60 & 0,1 & 2,04 & $0,85-4,9$ \\
\hline \multicolumn{6}{|c|}{ Sedentarismoc y edad ${ }^{d}$} \\
\hline Mujeres & 1,25 & 8,90 & 0,0003 & 3,50 & $1,50-7,88$ \\
\hline Hombres & 0,30 & 0,64 & 0,42 & 1,35 & $0,64-2,86$ \\
\hline
\end{tabular}

a Según Katz (16).

b Intervalo de confianza de $95 \%$.

${ }^{c}$ Sedentarismo es la inversa de la práctica de ejercicio físico: $s i ́=1$, no $=0$.

${ }^{d}$ La edad se consideró en escala dicotómica: de 30 a 49 años $=0 ; 50$ años o más $=1$.

19). Sin embargo, en la presente investigación no se encontró esa asociación, ya fuera de manera individual o combinada con otras variables.

Se observó una asociación estadísticamente significativa entre la HTA y los antecedentes familiares de HTA independientemente de las demás variables, lo que confirma el posible componente genético de esta afección (5, 21). No obstante, esta asociación podría deberse a otros factores subyacentes que solo se manifiestan al combinarse con determinadas circunstancias extrínsecas, como el sedentarismo y la alimentación inadecuada.

En total, 99 personas (35,3\%; IC95\%: 30 a 41\%) tenían sobrepeso (IMC > 27,3), cifra ligeramente superior al $27 \%$ encontrado en estudios previos realizados en México (7). Al igual que en esos estudios, el sobrepeso mostró una asociación muy significativa con la HTA, particularmente en mujeres $(4,7)$.

Se encontró una clara asociación inversa entre la práctica de ejercicio físico y la HTA, lo que corrobora el efecto protector del ejercicio encontrado por otros autores $(19,22)$. Se debe resaltar que las mujeres presentaron una menor prevalencia de HTA que los hombres y que practicaron el ejercicio físico con mayor frecuencia que ellos, tal vez debido a su mayor preocupación por mantener una figura esbelta o porque cuidan más de su salud que los hombres.

$\mathrm{Al}$ analizar la interacción mediante la regresión logística multifactorial se encontró que los factores asociados con la HTA fueron, en orden decreciente, el sexo masculino, los antecedentes familiares de HTA, el sobrepeso y el no practicar ejercicio físico. Sin embargo, esas asociaciones no fueron significatique estos mostraron una mayor tendencia a padecer de HTA.

Con el fin de verificar esta hipótesis se repitió el análisis de las variables estratificadas por el sexo y se confirmó que tanto la asociación individual de cada variable con la HTA como la de su combinación resultaron significativas solo en mujeres. Así, la asociación encontrada entre la edad y la HTA fue mayor en mujeres que en hombres, posiblemente debido al efecto protector del estrógeno. También los antecedentes familiares de HTA tuvieron una mayor asociación con la HTA en mujeres que en hombres, mientras que el sobrepeso y la práctica de ejercicio físico resultaron significativamente asociados con la HTA solamente en mujeres.

$\mathrm{Al}$ analizar la interacción de las variables señaladas, se encontró que únicamente en mujeres se observan interacciones directas significativas, por vas en los hombres, debido en parte a ejemplo, entre los antecedentes familiares de HTA y el sobrepeso, lo que puede indicar que el componente genético tiene mayores posibilidades de expresarse en mujeres con sobrepeso. También se encontró una interacción directa muy significativa entre el sedentarismo y la edad, lo que podría indicar que el sedentarismo eleva el riesgo de HTA en los grupos de mayor edad, particularmente en mujeres, ya que esa interacción no fue significativa en hombres.

Aunque se ha señalado que existe una interacción directa entre el sedentarismo y el sobrepeso en general, Arroyo y colaboradores no encontraron una asociación entre ambas variables conjuntas y la HTA, aunque sí observaron una asociación entre la obesidad y la HTA, especialmente en mujeres (7). Esos resultados coinciden con los obtenidos en la presente investigación.

En conjunto, todas estas interacciones parecen indicar que la HTA en hombres es un fenómeno cuya presencia es independiente de los factores de riesgo tradicionales como la edad, los antecedentes familiares, la obesidad y el sedentarismo y que pudiera existir un factor adicional asociado con el polimorfismo en el cromosoma Y (21). Esta posibilidad debe tomarse en cuenta para estudios epidemiológicos de la HTA y para planificar programas preventivos.

Se recomienda que los estudios de los factores de riesgo de HTA se centren preferentemente en adultos mayores de 30 años y que se tome en cuenta la práctica del ejercicio físico como variable de confusión. Mientras tanto, los programas de prevención de la HTA deben hacer mayor hincapié en reducir el peso y promover la actividad física, sobre todo en los hombres, quienes a pesar de ser más propensos a padecer de HTA, participan menos en los programas preventivos. Además de las recomendaciones alimentarias e higiénicas generales, se recomienda crear programas de diagnóstico temprano y tratamiento dirigidos a los hombres, ya que a diferencia de lo observado en las mujeres, la reducción de peso y la práctica de ejercicio físico en este grupo pueden ser insuficientes para reducir el riesgo de HTA. 


\section{REFERENCIAS}

1. Andersson $\mathrm{OK}$, Almgren T, Persson B, Samuelsson O, Hedner T, Wilhelmsen L. Survival in treated hypertension: follow up study after two decades. Br Med J. 1998;317:167-71.

2. Chalmers J, MacMahon S, Mancia G, Whitworth J, Beilin L, Hansson L, et al. 1999 World Health Organization-International Society of Hypertension guidelines for the management of hypertension. Clin Exp Hypertens. 1999;21: 1009-60.

3. Velázquez-Monroy $\mathrm{O}$, Rosas Peralta $\mathrm{M}$, Lara Esqueda A, Pastelín Hernández G, SánchezCastillo C, Attie F, et al. Prevalencia e interrelación de enfermedades crónicas no transmisibles y factores de riesgo cardiovascular en México. Resultados finales de la Encuesta Nacional de Salud 2000. Arch Cardiol Mex. 2002; 72:71-84.

4. Kannel WB, Zhang T, Garrison RJ. Is obesityrelated hypertension less of a cardiovascular risk? The Framingham Study. Am Heart J. 1990;120:1195-201.

5. Guémez Sandoval JC, Moreno Altamirano L, Kurí Morales P, Argote Roumagnere A, Alba Leonel A, Méndez Vargas R, et al. Estilos de vida, antecedentes familiares y personales patológicos en relación a la hipertensión arterial en estudiantes de la Facultad de Medicina de la UNAM. Arch Inst Cardiol Mex. 1990;60: 283-7.

6. Bautista LE, Vera-Cala LM, Villamil L, Silva SM, Pena IM, Luna LV. Factores de riesgo asociados con la prevalencia de hipertensión arterial en adultos de Bucaramanga, Colombia. Salud Publica Mex. 2002;44(5):399-405.

7. Arroyo P, Fernández V, Ávila-Rosas H. Overweight and hypertension: data from the 1992-
1993 Mexican survey. Hypertension. 1997;30: 646-9.

8. Hazuda HP. Hypertension in the San Antonio Heart Study and the Mexico City Diabetes Study: sociocultural correlates. Public Health Rep. 1996;111(Suppl2):18-21.

9. Burt VL, Cutler JA, Higgins M, Horan MJ, Labarthe D, Whelton $\mathrm{P}$, et al. Trends in the prevalence, awareness, treatment, and control of hypertension in the adult U.S. population. Data from the health examination surveys, 1960 to 1991. Hypertension. 1995;26:60-9.

10. Hori $Y$, Toyoshima H, Kondo T, Tamakoshi $\mathrm{K}$ Yatsuya H, Zhu S, et al. Gender and age differences in lifestyle factors related to hypertension in middle-aged civil service employees. J Epidemiol. 2003;13(1):38-47.

11. Sistema para la consulta de información censal (SCINCE). Datos del XII censo general de población y vivienda para el Estado de Colima año 2000. México, D.F.: Instituto Nacional de Estadística, Geografía e Informática; 2002.

12. McAlister FA, Straus SE. Evidence based treatment of hypertension. Measurement of blood pressure: an evidence based review. $\mathrm{Br}$ Med J. 2001;322(7291):908-11.

13. Aguirre-Ramos R, Trujillo-Hernández B, Huerta M, Trujillo X, Vásquez C, MillánGuerrero RO. White-coat hypertension and risk factors in recently diagnosed hypertensive patients. Gac Med Mex. 2002;138:319-24.

14. Burton BT, Foster WR, Hirsch J, Van Itallie TB. Health implications of obesity: an NIH consensus development conference. Int J Obes. 1985;9(3):155-70.
15. Hosmer DW, Lemeshow S. Applied logistic regression. New York: John Wiley \& Sons; 1989.

16. Katz MH. Multivariable analysis. Cambridge: Cambridge Univ Press; 1999.

17. González Villalpando C, Stern MP, Arredondo Pérez B, Valdez R, Mitchell B, Haffner $S$. Prevalence and detection of hypertension in Mexico. Arch Med Res. 1994;25:347-53.

18. Reich H, Duncan JA, Weinstein JC, Daniel C, Scholey JW, Miller JA. Interactions between gender and the angiotensin type 1 receptor gene polymorphism. Kidney Int. 2003;63: 1443-9.

19. Stamler J, Rose G, Elliot P, Dyer A, Marmot M, Kesteloot $\mathrm{H}$, et al. Findings of the International Cooperative INTERSALT Study. Hypertension. 1991;17(Suppl1):9-25.

20. Rehm J, Greenfield TK, Walsh G, Xie X, Robson L, Single E. Assessment methods for alcohol consumption, prevalence of high risk drinking and harm: a sensitivity analysis. Int J Epidemiol. 1999;28:219-24.

21. Sing CF, Boerwinkle E, Turner ST. Genetics of primary hypertension. Clin Exp Hypertens. 2000;8:623-51.

22. Whelton SP, Chin A, Xin X, He J. Effect of aerobic exercise on blood pressure: a metaanalysis of randomized, controlled trials. Ann Intern Med. 2002;136:493-503.

Manuscrito recibido el 20 de junio de 2003. Aceptado para publicación, tras revisión, el 29 de octubre de 2004.
ABSTRACT

\section{Analysis of risk factors for hypertension in Colima, Mexico}

Objective. To evaluate the possible association that age, sex, excess weight, family history of hypertension, alcoholism, and sedentary lifestyle have with hypertension in the adult population of the city of Colima, Mexico.

Methods. This was a population-based analytic cross-sectional study. A structured survey was used with 280 adults older than 30 years of age who were living in the city of Colima in 2001 and 2002. The variables studied were sex, age, weight, height, family history of hypertension, engaging in physical exercise, smoking, and consuming alcohol. Blood pressure (BP) was measured with the auscultatory method. Borderline or doubtful measurements were checked again four or five days later. Hypertension was defined as systolic $\mathrm{BP} \geq 140 \mathrm{~mm} \mathrm{Hg}$ and diastolic blood pressure $\geq 90 \mathrm{~mm} \mathrm{Hg}$, or as the person being under antihypertensive treatment. The odds ratios (ORs) of the variables studied were calculated, along with their $95 \%$ confidence intervals (95\% CIs). The association between the variables and hypertension was estimated through logistic regression, and their interaction through the coefficient of the interaction products.

Results. The overall prevalence of hypertension was $28.6 \%$. The prevalence was higher in men than in women ( $42.1 \%$ vs. $19.2 \%$; OR $=3.04,95 \%$ CI: 1.8 to 5.2$)$ and in people older than 49 years than in people 30 to 49 years old ( $36.8 \%$ vs. $21.9 \%$; OR $=2.07,95 \%$ CI: 1.22 to 3.50). A family history of hypertension and excess weight were associated with hypertension, while physical exercise had a protective effect $(\mathrm{OR}=0.45 ; 95 \% \mathrm{CI}: 0.23$ to 0.86 ). There was interaction between hypertension and age $\geq 50$ years, a family history of hypertension, overweight, and physical exercise, especially among women.

Conclusions. The prevalence of hypertension in Colima is very similar to that for Mexico as a whole. The strong association that hypertension had with male gender, regardless of the other variables, emphasizes the need for promoting prevention campaigns that focus more on men. 